\title{
Ocular abnormalities and complications in anterior megalophthalmos: a case series
}

\author{
Devesh Kumawat $^{1} \cdot$ Tanveer Alam $^{1} \cdot$ Pranita Sahay $^{1} \cdot$ Rohan Chawla $^{1}$
}

Received: 17 June 2018 / Revised: 2 December 2018 / Accepted: 12 December 2018 / Published online: 7 January 2019

(c) The Royal College of Ophthalmologists 2019

\begin{abstract}
Objectives To describe the clinical and Scheimpflug imaging features in cases of anterior megalophthalmos (AM). Methods Retrospective record review was performed for patients with AM who presented between June 2017 and May 2018. Clinical history, slit lamp examination, Scheimpflug imaging indices (Pentacam-HR, Oculus, GmbH), dilated fundus examination and treatment records were reviewed.

Results The study included eight eyes of four male patients (mean age 6.5 years, range 4-10 years). Corrected distance visual acuity ranged from finger counting to 20/80. The mean horizontal corneal diameter, central corneal thickness, steep keratometry, flat keratometry, anterior chamber (AC) angle, AC depth, maximum pupil diameter and axial length were 13.8 $\pm 0.5 \mathrm{~mm}, 538.7 \pm 68.9 \mu \mathrm{m}, 42.8 \pm 1.6 \mathrm{D}, 41.4 \pm 0.9 \mathrm{D}, 47.0 \pm 4.2$ degree, $3.8 \pm 0.3 \mathrm{~mm}, 3.9 \pm 0.1 \mathrm{~mm}$, and $24.9 \pm 0.9 \mathrm{~mm}$, respectively. Posterior dislocation of crystalline lens, vitreous degeneration and rhegmatogenous retinal detachment (consequent of retinal dialysis/atrophic hole/lattice with hole) were noted in seven, eight and five eyes, respectively. Pigment dispersion glaucoma was noted in both eyes of one patient, which was managed with topical anti-glaucoma medication. Vitrectomy with silicone oil tamponade was successful in retinal reattachment in all three eyes that underwent surgery. Conclusion Scheimpflug imaging helps in demonstrating the corneal and anterior segment pathological changes in AM. The disease extends to involve the zonules, vitreous and retina as well. Ophthalmologists should be able to identify this disorder, recognise and manage the associations and complications.
\end{abstract}

\section{Introduction}

Anterior megalophthalmos (AM) is an uncommon disorder of congenital stationary enlargement of the anterior segment of the eye [1-3]. It is characterised by a clear enlarged cornea (megalocornea) with a horizontal corneal diameter (HCD) of $13 \mathrm{~mm}$ or more, deep anterior chamber (AC), iris hypoplasia, enlarged ciliary body ring, subluxation or dislocation of lens and absence of features suggestive of congenital glaucoma. It probably results from keratodysgenesis and/or iridogoniodysgenesis [1]. Keratoglobus

Supplementary information The online version of this article (https:// doi.org/10.1038/s41433-018-0329-3) contains supplementary material, which is available to authorized users.

Rohan Chawla

dr.rohanrpc@gmail.com

1 Dr Rajendra Prasad Centre for Ophthalmic Sciences, All India Institute of Medical Sciences, New Delhi, India and buphthalmos due to congenital glaucoma are its close differentials [1, 3, 4].

Enough literature exists on morphological types of cataract, cataract surgery and its complications in adult patients with AM [5]. The posterior segment changes in the form of vitreo-retinopathy and pigmentary glaucoma are scarcely reported in AM [1, 3, 6, 7]. Scheimpflug imaging (SI) in a couple of cases of AM has also been reported [8, 9]. However, none provides a comprehensive ophthalmic evaluation. This study reports the clinical features, SI features and posterior segment abnormalities in eight eyes of four paediatric patients.

\section{Materials and methods}

A retrospective review of records of patients with AM who presented between June 2017 and May 2018 was performed. The study adhered to the tenets of Declaration of Helsinki. Institute Ethics Committee approval was obtained. The diagnosis of AM was made as per the standard criteria [1, 3]. 
Detailed history, general physical examination, slit lamp examination, SI (Pentacam-HR, Oculus GmbH, Wetzlar, Germany) and dilated fundus examination records were reviewed. The data were entered into a Excel sheet and analysed using the STATA SE 12.1 software.

\section{Results}

The study included eight eyes of four male patients. The major clinical features and SI parameters are mentioned in Tables 1 and 2, respectively. The individual case details are as follows:

\section{Case 1}

A 4-year-old boy was brought by parents with the complaint of poor vision in both eyes and large eyes since birth. Parents did not give a history of birth injury, watering and photophobia. There were no systemic complaints.

The unaided distance visual acuity (UDVA) was finger counting (FC) close to face and FC at $2 \mathrm{~m}$ in right and left eye, respectively. Slit lamp examination revealed bilateral symmetrical large clear corneas with no evidence of Descemet membrane tears, well-defined corneo-scleral junction, few pigments on corneal endothelium, deep AC, iridodonesis, diffuse iris atrophy, aphakia and opticaly empty anterior vitreous cavity with fibrillar vitreous strands (Fig. 1a, b). The pupils dilated poorly with a topical tropicamidephenylephrine application. The fundus examination showed posterior dislocated crystalline lens in both eyes (Fig. 1c, d). The optic discs were large in both eyes with a cup-disc ratio (CDR) being 0.5:1 and 0.6:1 in right and left eye, respectively. The right eye had total rhegmatogenous retinal detachment (RRD) with few subretinal bands and no identifiable retinal break/lattice degeneration/meridional folds. The left eye retina was unremarkable. The corrected distance visual acuity (CDVA) in the left eye was 20/80 as evaluated on Snellen E chart.

The SI findings were as follows: HCD right eye or oculus dexter (OD) $14.4 \mathrm{~mm}$, left eye or oculus sinister (OS) 14.5 $\mathrm{mm}$; steep keratometry OD $41.2 \mathrm{D}$, OS $40.8 \mathrm{D}$; flat keratometry OD $41.1 \mathrm{D}$, OS $40.1 \mathrm{D}$. The four map refractive analysis of the left eye is shown in Supplemental Figure. 1. The specular endothelial count was 2987 and 2910 cells/ $\mathrm{mm}^{2}$ in right and left eye, respectively. The axial length was 24.2 and $24.7 \mathrm{~mm}$ in the right and left eye, respectively. The Goldmann applanation intraocular pressure (IOP) was 10 and $14 \mathrm{mmHg}$ in right and left eye, respectively. A diagnosis of bilateral AM with vitreo-retinopathy with OD RRD was made.

The child was taken for right eye encirclage, pars plana vitrectomy (PPV) and silicone oil tamponade. The

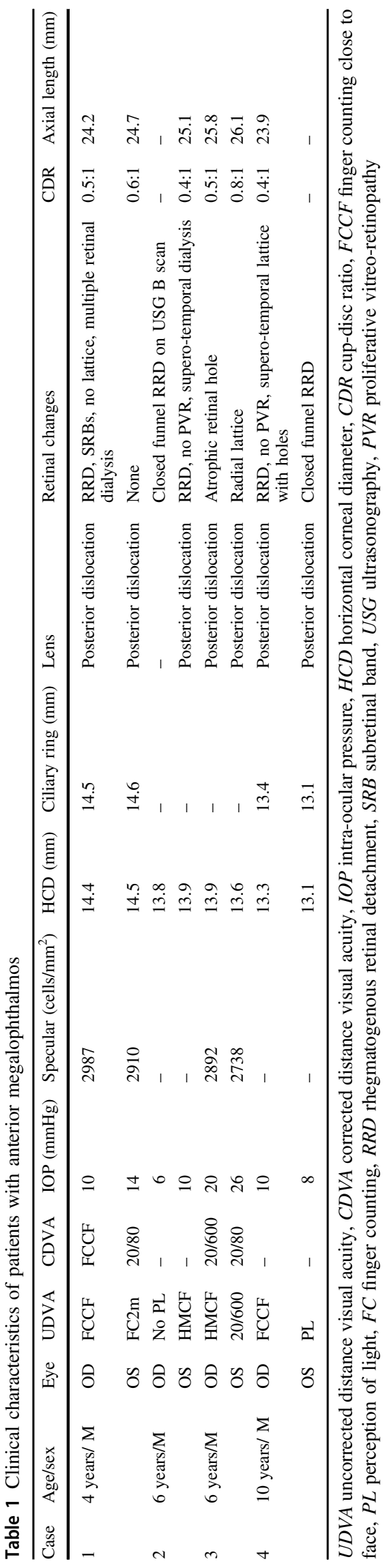


Table 2 Scheimpflug imaging characteristics of eyes with anterior megalophthalmos

\begin{tabular}{lllllllll}
\hline Case & Eye & Steep/flat K $(\mathrm{D})$ & CCT $(\mu \mathrm{m})$ & TCT $(\mu \mathrm{m})$ & PE $(\mu \mathrm{m})$ & ACD $(\mathrm{mm})$ & ACA $(\mathrm{deg})$ & PD $(\mathrm{mm})$ \\
\hline 1 & OD & $41.2 / 40.8$ & 644 & 630 & +4 & 3.7 & 48.0 & 4.2 \\
& OS & $41.1 / 40.1$ & 631 & 619 & +3 & 3.8 & 48.9 & 4.0 \\
2 & OD & $41.4 / 40.6$ & - & - & - & - & - & - \\
& OS & $41.6 / 41.1$ & 528 & 498 & +5 & 4.4 & 49.7 & 4.1 \\
3 & OD & $44.3 / 42.2$ & 495 & 485 & -3 & 3.8 & 50.8 & 3.8 \\
& OS & $43.2 / 41.4$ & 489 & 475 & -2 & 3.9 & 49.5 & 3.7 \\
4 & OD & $44.6 / 42.5$ & 490 & 479 & -1 & 3.4 & 41.6 & 4.0 \\
& OS & $44.8 / 42.6$ & 494 & 488 & -2 & 3.5 & 40.5 & 4.0 \\
\hline
\end{tabular}

$C C T$ central corneal thickness, TCT thinnest corneal thickness, $P E$ posterior elevation, $A C D$ anterior chamber depth, $A C A$ anterior chamber angle, $P D$ pupil diameter preoperative ultrasound biomicroscopy $(35 \mathrm{MHz})$ revealed a deep AC, normal flat iris root insertion and widened ciliary body ring (OD $14.5 \mathrm{~mm}$, OS $14.6 \mathrm{~mm}$ ). The UBM of the left eye is shown in Supplemental Figure. 2. Multiple retinal dialysis were noted in the right eye in the absence of flap tears. The retina appeared to be disinserting at the ora at multiple locations. The left eye retinal examination under anaesthesia did not reveal any known peripheral treatable lesion. However, a $360^{\circ}$ laser barrage (four rows) was done at the ora prophylactically. At the end of 6 weeks post surgery, the retina was attached in the right eye with an IOP of $12 \mathrm{mmHg}$ and a CDVA of 20/200.

The pedigree analysis showed a non-consanguineous marriage with similar findings in an elder sibling (case 2).

\section{Case 2}

A 6-year-old boy was brought by parents with complaints of loss of vision in right eye for a few months and diminution of vision in the left eye for few days. There were no systemic complaints.

The UDVA in the right and left eye was no perception of light and hand movements close to face, respectively. There were bilateral symmetrical large clear corneas. The right eye had total hyphaema and a closed funnel retinal detachment on ultrasonography (USG) B scan. The left eye had poorly dilating pupil, aphakia, an optically empty vitreous cavity with vitreous strands, posterior dislocation of the lens and a total RRD with no proliferative vitreo-retinopathy (PVR). The IOP in right and left eye were 6 and $10 \mathrm{mmHg}$, respectively. The HCD was 13.8 and $13.9 \mathrm{~mm}$ in right and left eye, respectively.

Left eye encirclage, PPV and silicone oil tamponade was performed. Retinal dialysis was noted in the superotemporal quadrant in the absence of flap tears. Six months post surgery, the retina remained attached with a corrected visual acuity of 20/120, an IOP of $18 \mathrm{mmHg}$ on topical timolol maleate $0.5 \%$ b.i.d. and a CDR of $0.4: 1$. The axial length in the left eye was $25.1 \mathrm{~mm}$ on optical biometry. The child awaits silicone oil removal in the left eye.

\section{Case 3}

A 6-year-old boy was brought by parents with complaints of gradually progressive diminution of vision in both eyes, more so in the right eye. Parents did not give a history of birth injury, watering and photophobia. The UDVA in right and left eye was hand movement close to face and 20/600, respectively. The slit lamp examination was similar to that in case 1 (Fig. 2a, b). Trans-illumination iris defects were seen in the left eye (Fig. 2b). A note was made of posterior dislocation of a clear crystalline lens in both eyes (Fig. 2c, d). The retina was attached in both eyes with an atrophic hole in the temporal periphery in the right eye and equatorial pigmented radial lattice degeneration in the left eye (Fig. 2c, d). The IOP in right and left eye were 20 and $26 \mathrm{mmHg}$, respectively. The CDR in right and left eyes was $0.5: 1$ and $0.8: 1$, respectively. The child was not cooperative for gonioscopy and visual field analysis. The CDVA in the right and left eye was $20 / 600$ and 20/80, respectively. The HCD was 13.9 and $13.6 \mathrm{~mm}$ in the right and left eye, respectively. The axial length was 25.8 and $26.1 \mathrm{~mm}$ in the right and left eye, respectively.

There were no systemic complaints. The family history was non-contributory. The habitus was normal. Cardiology evaluation and echocardiography turned out to be normal. A diagnosis of bilateral AM with secondary glaucoma was made. Laser delimitation of the retinal hole and lattice degeneration was performed in the right and left eye, respectively. Topical timolol maleate-brimonidine combination therapy was started in both eyes. One month after starting the medication, the IOP was 12 and $14 \mathrm{mmHg}$ in the right and left eye, respectively.

\section{Case 4}

A 10-year-old boy presented with complaints of sudden onset diminution of vision in the right eye for past 3 weeks. The left eye had poor vision for 2 years. The UDVA in the right and left eye was FC close to face and perception of light, respectively. The slit lamp examination was similar to 
Fig. 1 Slit lamp colour photograph of the anterior segment of right and left eye of a 4 -year-old boy with anterior megalophthalmos (case 1) shows large clear corneas, welldefined corneo-scleral junction, diffuse iris atrophy and aphakia $(\mathbf{a}, \mathbf{b})$. Ultra-wide field pseudocolour fundus image of the right eye (c) shows a posteriorly dislocated crystalline lens and a total rhegmatogenous retinal detachment with few subretinal bands along the supero-temporal arcade and in temporal midperiphery. A posteriorly dislocated clear crystalline lens was noted in the left eye as well (d). The left eye retinal examination was unremarkable
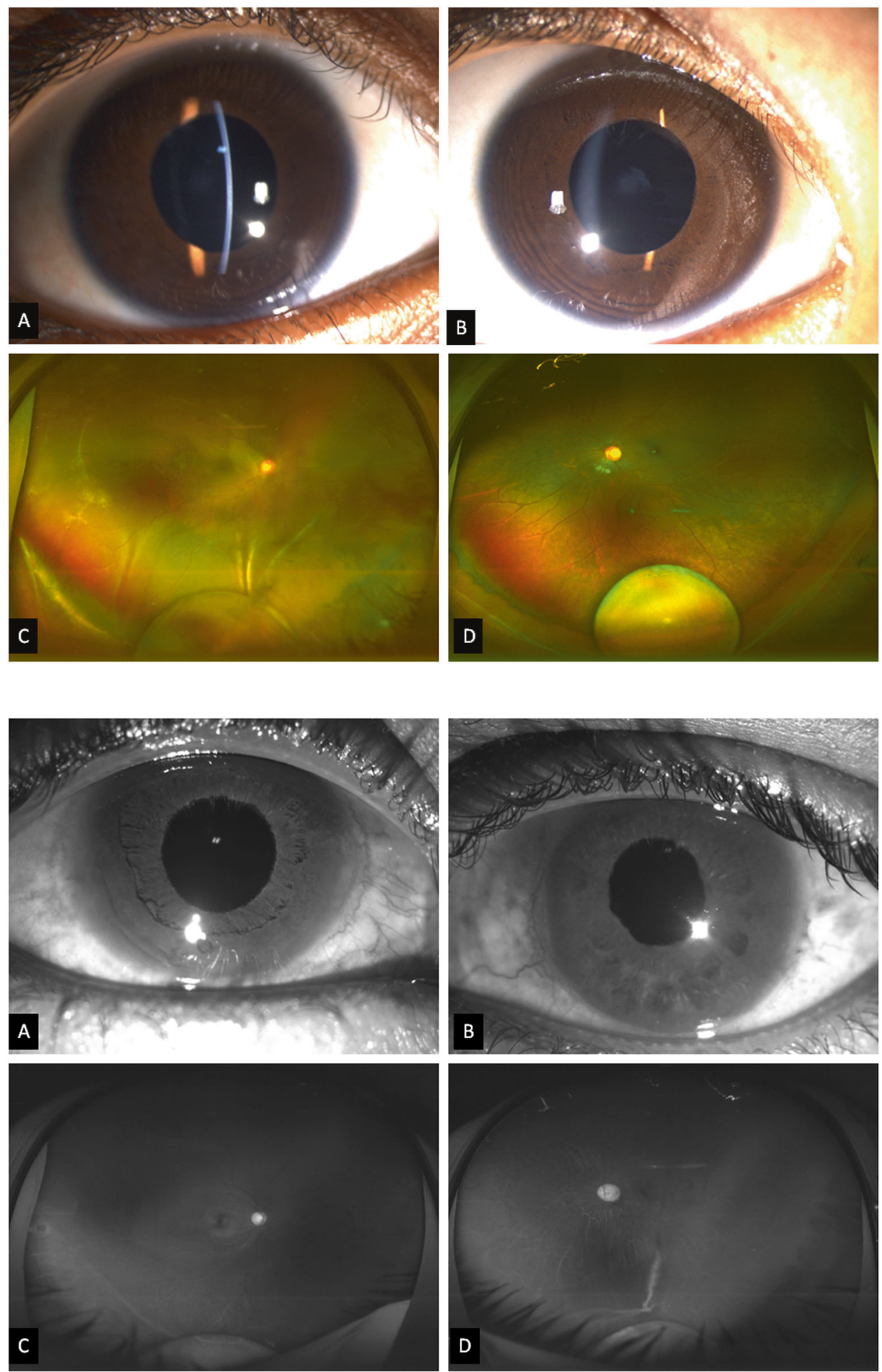

Fig. 2 Slit lamp photograph of the anterior segment of right and left eye of a 6-year-old boy with anterior megalophthalmos (case $3)$ shows large clear corneas, well-defined corneo-scleral junction, diffuse iris atrophy and aphakia $(\mathbf{a}, \mathbf{b})$. In addition, transillumination defects are seen in the inferior half of iris in the left eye (b). Ultra-wide field fundus images show posteriorly dislocated crystalline lens and attached retina in both eyes with an atrophic hole in the temporal periphery in the right eye (c) and inferior equatorial radial lattice degeneration in the left eye (d). Glaucomatous cupping can be noted in both eyes, more so in the left eye that in case 1. A note was made of posterior dislocation of the crystalline lens in both eyes, total RRD with no identifiable break/lattice degeneration and no PVR in the right eye and closed funnel RRD in the left eye. The CDR in the right eye was $0.4: 1$. There was no family history of such complaints. The systemic examination was unremarkable as in case 3 . The HCD was 13.3 and $13.1 \mathrm{~mm}$ in the right and left eye, respectively.

Right eye encirclage, PPV and silicone oil tamponade was performed. The preoperative UBM $(35 \mathrm{MHz})$ revealed normal iris root insertion and widened ciliary body ring (OD $13.4 \mathrm{~mm}$, OS $13.0 \mathrm{~mm}$ ). A supero-temporal lattice with 
a hole and an inferior lattice were noted. At 4 weeks followup, the retina was attached in the right eye with a CDVA of $20 / 300$ and an IOP of $16 \mathrm{mmHg}$.

\section{Discussion}

$\mathrm{AM}$ is an entity distinct from simple megalocornea [1]. Simple megalocornea has enlarged clear cornea of normal thickness, normal anterior segment and normal intraocular pressure. Apart from megalocornea, AM has additional anterior segment changes in the form of widened ciliary ring leading to a deep AC, iris hypoplasia, zonular weakness, angle abnormalities and subluxation/ dislocation of lens [1].

The current study demonstrates that the pathological features in AM are not limited to the cornea and anterior segment, but commonly extends to involve the zonules, vitreous and retina as well. Spontaneous posterior dislocation of clear crystalline lens, vitreous degeneration and RRD were noted in seven, six and five eyes, respectively. In addition, SI features are also described in detail for these four patients.

All four patients were male and this supports the prevalent opinion that the inheritance pattern is mostly $\mathrm{X}$ linked recessive [1, 2]. However, Ahmadieh et al. [3] and certain other authors have reported equal distribution among females as well and thereby other modes such as autosomal form can also exist [6]. Axial myopia (axial length $>24.5$ $\mathrm{mm}$ ) was noted in four out of six eyes in our series. Axial myopia is frequently reported in $\mathrm{AM}$ and presence of an overall larger size eye does not contradict a diagnosis of AM $[1,3,10]$.

Vail et al. [1] first described this entity in middle-aged and elderly population. Thereafter, only a couple of case reports and a single series $(n=4)$ were reported in the paediatric population $[2,3,11]$. The age range at the time of development of RD was 8-34 years (mean 28 years) and 28-49 years in large series by Ahmadieh et al. [3] and Dufour et al. [12], respectively. All four patients in our study were below ten years. The development of characteristic changes (both anterior and posterior segment) at such an early age supports the fact that the zonular and vitreoretinal degeneration begin very early in childhood. The risk of form vision deprivation amblyopia becomes very high in such eyes. Even in three eyes of our series with unremarkable macula, the best corrected visual acuity was not $>20 / 80$ perhaps due to ammetropic amblyopia. The age of presentation was lower in our cases as compared to previous literature $[3,7,11]$, perhaps due to a greater referral of paediatric vitreoretinal cases to our tertiary care referral centre.

The Scheimpflug camera provides high-resolution images with accurate anterior and posterior corneal surface data, AC angle and depth, and lenticular thickness and density [13]. The cornea in our series was neither abnormally flat nor steep, with the steep keratometry ranging between 41.1 and $44.8 \mathrm{D}$. The corneal thickness was normal to slightly thin (considering a normal mean value of $520 \mu \mathrm{m})$ in most eyes except in case 1 where it exceeded $600 \mu \mathrm{m}$. A single case report on thinner cornea has been reported where the central pachymetry was $390 \mu \mathrm{m}$ [8]. Thicker cornea in AM has also been reported previously where the CCT was around $630 \mu \mathrm{m}$ [7]. None of the eyes had significant posterior elevation in our series, ruling out the possibility of corneal ectasia. On comparing the AC angle of healthy adults (with open-angle) on Scheimpflug camera to our cases, we found the angle to be larger in seven out of the eight eyes studies by us [14]. The maximum pupil diameter was consistently around $4 \mathrm{~mm}$. Poor pupillary dilatation in these eyes occurs due to dilator iris muscle hypoplasia or atrophy. These anterior segment findings prove very useful while considering buphthalmos and keratoglobus as the differentials.

AM and buphthalmos due to congenital glaucoma are close differentials of each other. A detailed history and corneal examination could help differentiate between the two $[1,3,15,16]$. While buphthalmos is associated with progressive enlargement of the cornea, Haab's striae, stretched out limbus, normal but high iris insertion pattern, increased IOP and glaucomatous disc damage; AM has nonprogressive congenital enlargement of the cornea, welldefined limbal anatomy, diffuse iris hypoplasia and usual absence of raised IOP and disc changes. However, secondary glaucoma can develop rarely early in life due to associated goniodysgenesis [1] and frequently later in life due to pigment dispersion $[3,6,10]$. One of our patients also had dispersed pigments in the AC and raised IOP with disc damage suggestive of pigment dispersion glaucoma. Coexistent glaucoma can worsen the visual outcome in AM eyes. Marfan syndrome may also be considered a close differential of AM as megalocornea, iris atrophy, poor pupillary dilation, glaucoma and vitreo-retinopathy occurs in Marfan syndrome as well [17]. However, patients with Marfan syndrome present late (usually in third- fourth decade), have high axial myopia and absence of differential anterior segment and ciliary enlargement [17].

Posterior dislocation of the clear crystalline lens was noted in all except one eye in which posterior segment could not be visualised. In cases of AM, the enlargement of ciliary ring seems to severely affect the zonular integrity. An inherent structural abnormality of the zonules is also a possibility. It is interesting to note that three of our patients presented with retinal detachment and did not have any prior handicap. Two cases had RRD in one eye for quite a long time and the patients may have not noticed unilateral loss of vision. When the fellow eye developed RRD and the 
patients had complete bilateral visual loss, it was then that the parents brought the cases to us. It may be possible that the dislocation was long-standing in all these cases. The dislocation of the lens would have had a significant effect on the vision only if the eye would have been sighted when it happened. The definite timing of dislocation/ RRD cannot be reliably judged based on the history as well as examination findings in these cases.

In a large case series of 46 eyes by Ahmadieh et al. [3], different vitreoretinal abnormalities were noted in AM namely vitreous syneresis and synchysis, cobblestone degeneration, white without pressure, lattice degeneration $(10.8 \%)$ and RRD (37.5\%). The RRD was spontaneous in all except two eyes where it was post traumatic. The existing literature suggests that the RRD in AM eyes occurs either spontaneously or after trauma/surgery (intracapsular or extracapsular cataract extraction) [3, 12, 18]. In our study, all eyes had a spontaneous development of RRD.

In eyes with relatively acute detachments, the retinal breaks could not be identified preoperatively. The breaks noted intra-operatively were all degenerative (either dialysis or atrophic hole or lattice with hole). The majority of breaks noted in previously reported series were horseshoe tears, which could have been due to a posterior vitreous detachment (PVD) in the described older patients. The younger the patient, lesser is the likelihood of PVD. The presence of multiple retinal dialysis as seen in case 1 has also been previously reported in a single case by Ahmadieh et al [3].

Analysing all the anterior and posterior segment abnormalities seen in AM in our study and existing literature [3, 19], we hypothesise that an anomaly in the development of the ciliary body region (pars plana and para plicata) leading to an enlargement of the ciliary body ring might be the primary abnormality in cases of AM. Consequent to this, the cornea enlarges and AC deepens. Additionally, an enlargement of the posterior segment ensues with abnormal retinal insertion at the pars plana, degenerative changes in the peripheral retina and accelerated vitreous syneresis and synchysis. However, there exist certain cases of AM who do not develop vitreo-retinopathy and glaucoma. These may represent a different benign morphological type of AM. Since we evaluated cases who presented to our centre with complaint of visual loss (from dislocation of lens or retinal detachment), the benign cases may have been missed due to the selection bias. Ahmadieh et al. [3] also reported vitreo-retinopathy in majority of their patients (up to three-quarters), thereby indicating that the benign form may be less prevalent. A prospective study with comparison of the SI and biometry between these two types may provide better insight into the disease pathophysiology.

The number of retinal breaks was few and thereby the PVR was relatively absent in our cases. Closed funnel RD noted in two eyes could be due to the detachments being very long standing. No surgical intervention could be performed in these eyes in view of very poor visual prognosis. The surgical success rate in the previous series range from 66. to $88.9 \%$ [3, 12]. Retinal reattachment was noted in all three eyes in our series. However, a longer follow-up following silicone oil removal will be required to comment on the final attachment rates.

To conclude, AM is probably a developmental disorder of the ciliary body region with secondary changes in both the anterior and posterior segment. Ophthalmologists should be able to identify this disorder, differentiate it from buphthalmos/keratoglobus, and recognise the associations and complications. A comprehensive management of existing glaucoma, lenticular abnormalities and vitreoretinopathy is required for optimal visual outcomes and long-term favourable prognosis.

\section{Summary Table}

\section{What was known before}

- $\mathrm{AM}$ is an uncommon disorder of congenital stationary enlargement of the anterior segment of the eye.

- AM, buphthalmos due to congenital glaucoma and keratoglobus are close differentials of each other.

\section{What this study adds}

- AM is probably a developmental disorder of the ciliary body region with secondary changes in both the anterior and posterior segment.

- Co-existing lenticular abnormalities, vitreo-retinopathy and glaucoma require a comprehensive management.

\section{Compliance with ethical standards}

Conflict of interest The authors declare that they have no conflict of interest.

Publisher's note: Springer Nature remains neutral with regard to jurisdictional claims in published maps and institutional affiliations.

\section{References}

1. Vail DT. Adult hereditary anterior megalophthalmus sine glaucoma: a definite disease entity: with special reference to the extraction of cataract. Arch Ophthalmol. 1931;6:39-62.

2. Zeiter HJ. Hereditary anterior megalophthalmos; the pedigree of two siblings. Am J Ophthalmol. 1964;57:315-7.

3. Ahmadieh H, Banaee T, Javadi MA, Jafarinasab MR, Yazdani S, Sajjadi H. Vitreoretinal disorders in anterior megalophthalmos. Jpn J Ophthalmol. 2006;50:515-23. 
4. Gupta N, Ganger A. Keratoglobus: a close entity to megalophthalmos. 2016;5:634.

5. Galvis V, Tello A, M Rangel C. Cataract surgery in anterior megalophthalmos: a review. Med Hypothesis Discov Innov Ophthalmol. 2015;4:101-8.

6. Turaçli ME, Tekeli O. Anterior megalophthalmos with pigmentary glaucoma. Graefes Arch Clin Exp Ophthalmol. 2005;243:1066-8.

7. Rao A, Videkar C. Hereditary anterior megalophthalmos with posterior vitreoretinopathy: a surgical challenge. BMJ Case Rep. 2014;2014:bcr2013202212. Published 2014 Mar 14.

8. Nemeth G, Hassan Z, Berta A, Modis L. Scheimpflug imaging in anterior megalophthalmos. Indian J Ophthalmol. 2013;61:32-35.

9. Nawani N, Jain AK, Singh R. Ultrasound biomicroscopy and scheimpflug imaging in anterior megalophthalmos: changes seen after cataract surgery. Case Rep Ophthalmol Med. 2015;2015:195950.

10. Huang C-X, Zhao X-H, Xing Y-Q. Combined phacoemulsification and anterior vitrectomy in a case of anterior megalophthalmos with open-angle glaucoma and high myopia. Int J Ophthalmol. 2017;10:1178-80.

11. Matalia JH, Tejwani S, Rajput VK, Matalia H. Small lens for a big eye: successful management of anterior megalophthalmos. Indian J Ophthalmol. 2018;66:457-9.
12. Dufour R, Scouras J, Gailloud CL. Megalophtalmie, subluxation cristallinienne et cataracte; decollement retinien. Ophthalmologica. 1972;165:409-16.

13. Belin MW, Ambrósio R. Scheimpflug imaging for keratoconus and ectatic disease. Indian J Ophthalmol. 2013;61:401-6.

14. Yi J-H, Lee H, Hong S, Seong GJ, Kang SY, Ma KT, et al. Anterior chamber measurements by pentacam and AS-OCT in eyes with normal open angles. Korean J Ophthalmol. 2008;22:242-5.

15. Meire FM, Delleman JW. Biometry in X linked megalocornea: pathognomonic findings. Br J Ophthalmol. 1994;78:781-5.

16. Ho CL, Walton DS. Primary megalocornea: clinical features for differentiation from infantile glaucoma. J Pediatr Ophthalmol Strabismus. 2004;41:11-17. quiz 46-47.

17. Cross HE, Jensen AD. Ocular manifestations in the Marfan syndrome and homocystinuria. Am J Ophthalmol. 1973;75:405-20.

18. Guixeres Esteve MC, Pardo Saiz AO, Martínez-Costa L, GonzálezOcampo Dorta S, Sanz Solana P. Surgical management of a patient with anterior megalophthalmos, lens subluxation, and a high risk of retinal detachment. Case Rep Ophthalmol. 2017;8:61-66.

19. Kuchenbecker J, Behrens-Baumann W. Ciliary body dysplasia in megalophthalmos anterior diagnosed using ultrasound biomicroscopy. Eye (Lond). 2002;16:638-9. 